\title{
Key factors influencing allied health research capacity in a large Australian metropolitan health district
}

\author{
Jennifer A Alison ${ }^{1,2}$ \\ Bill Zafiropoulos 1,2 \\ Robert Heard ${ }^{3}$
}

'Faculty of Health Sciences Discipline of Physiotherapy, University of Sydney, ${ }^{2}$ Allied Health Professorial Unit, Sydney Local Health District, ${ }^{3}$ Faculty of Health Sciences, Discipline of Behavioral and Social Sciences in Health, University of Sydney, Sydney, NSW, Australia
Correspondence: Jennifer A Alison Faculty of Health Sciences Discipline of Physiotherapy, University of Sydney, Cumberland Campus, PO Box 170 , Lidcombe, Sydney, NSW 1825, Australia Tel +6I 2 935। 937।

Fax +6I 2 935| $960 \mid$

Email jennifer.alison@sydney.edu.au
This article was published in the following Dove Press journal:

Journal of Multidisciplinary Healthcare

9 August 2017

Number of times this article has been viewed

Objective: The aim of this study was to identify key factors affecting research capacity and engagement of allied health professionals working in a large metropolitan health service. Identifying such factors will assist in determining strategies for building research capacity in allied health.

Materials and methods: A total of 276 allied health professionals working within the Sydney Local Health District (SLHD) completed the Research Capacity in Context Tool (RCCT) that measures research capacity and culture across three domains: organization, team, and individual. An exploratory factor analysis was undertaken to identify common themes within each of these domains. Correlations were performed between demographic variables and the identified factors to determine possible relationships.

Results: Research capacity and culture success/skill levels were reported to be higher within the organization and team domains compared to the individual domain (median [interquartile range, IQR] 6 [5-8], 6 [5-8], 5 [3-7], respectively; Friedman $\left.\chi^{2}(2)=42.04, p<0.001\right)$. Exploratory factor analyses were performed to identify factors that were perceived by allied health respondents to affect research capacity. Factors identified within the organization domain were infrastructure for research (eg, funds and equipment) and research culture (eg, senior manager's support for research); within the team domain the factors were research orientation (eg, dissemination of results at research seminars) and research support (eg, providing staff research training). Within the individual domain, only one factor was identified which was the research skill of the individual (eg, literature evaluation, submitting ethics applications and data analysis, and writing for publication).

Conclusion: The reported skill/success levels in research were lower for the individual domain compared to the organization or team domains. Key factors were identified in each domain that impacted on allied health research capacity. As these factors were different in each domain, various strategies may be required at the level of the organization, team, and individual to support and build allied health research capacity.

Keywords: research culture, organization, team, individual

\section{Introduction}

In the current health care climate, health care interventions and models of delivery are frequently being challenged to demonstrate their efficiency and cost-effectiveness. ${ }^{1}$ There is an increased expectation for allied health professionals to prove their value by providing evidence of the effectiveness of their interventions. ${ }^{1,2}$ Research in a clinical setting carried out by health care professionals involved in patient care is considered to be important in improving overall patient outcomes and optimizing health service efficiencies and cost-effectiveness..$^{2-4}$ Furthermore, there is evidence that research-active 
clinicians are more likely to translate new evidence into clinical practice. ${ }^{2,5,6}$ However, clinician engagement in research often results in conflicting time demands between clinical patient load and research productivity. ${ }^{2,5,7}$ The performance of research in the clinical setting by allied health professionals is therefore often perceived as difficult and may be viewed as a luxury. A positive research culture within health care organizations may facilitate evidence-based clinical practice and support clinical staff to build research capacity, which can drive changes in service delivery and health care policies. ${ }^{1,6,8-10}$

Research culture is regarded as an environment within an organization that enables and supports research to generate new knowledge and opportunities to translate evidence into practice. ${ }^{11,12}$ An enabling research culture is regarded as essential to building research capacity. ${ }^{11-14}$ Research capacity is the ability to carry out and produce research, whereas research capacity building describes a process of individual or institutional development which leads to a higher level of skill, with a greater ability to perform research that promotes the health of individuals and communities. ${ }^{1}$ It has been suggested that a whole-of-organization approach to building research capacity is imperative in reducing barriers to research engagement ${ }^{13,15,16}$ since the culture of an organization in which teams and individuals work will influence their abilities and opportunities to perform research. ${ }^{8,13}$

Studies that have investigated research culture and research capacity have either evaluated health professions grouped together (such as medicine, nursing, and allied health), ${ }^{7,17}$ or allied health professions collectively, ${ }^{6,12,13,18}$ or individual allied health disciplines separately (such as physiotherapy, ${ }^{2,5}$ speech pathology, ${ }^{19,20}$ occupational therapy, ${ }^{21}$ social work, ${ }^{22}$ nutrition and dietetics, ${ }^{23}$ podiatry, ${ }^{24,25}$ and radiation therapy ${ }^{26}$ ). Some of the factors identified as enablers of research capacity building and culture included provision of organizational structures that support research, having a research facilitator or mentor, having dedicated research positions, having research incorporated into job descriptions, encouraging collaborations with external partners such as universities, and enabling individuals to access research training and resources. ${ }^{6,7,12,13,17,21-23,25}$ Studies that specifically investigated factors affecting research culture and capacity in allied health professionals ${ }^{6,7,12,17-27}$ have used a variety of quantitative and qualitative statistical methods and have utilized focus groups, interviews, and validated and nonvalidated self-designed questionnaires which have made overall interpretation of these results difficult. A number of these studies ${ }^{6,7,12,17,18,23-25}$ used the Research Capacity in
Context Tool (RCCT), ${ }^{18}$ which is a validated survey that measures indicators of research capacity and culture at an organizational, team, and individual level. Since research capacity building should involve a whole system approach with strategies directly targeting the organization, team, and individual levels, ${ }^{6,12,13}$ this tool may assist in highlighting specific areas that can be targeted within these domains.

The primary aim of the study was to identify key factors at the organization, team, and individual levels that affect research capacity of allied health professionals working in a large metropolitan health service. The secondary aim was to determine whether there were any relationships between the demographic characteristics of the study sample and the key factors affecting research capacity.

\section{Materials and methods}

The study design was a prospective survey. Ethics approval was obtained from the Human Research Ethics Committee of the Sydney Local Health District (SLHD; Protocol Number X15-0240 and LNR/15/RPAH321).

\section{Sample}

The sample was recruited from allied health professionals working in the SLHD. SLHD is located in the center and inner west of Sydney and provides health care services to more than 615,790 people over a land area of approximately $126 \mathrm{~km}^{2}$ in Sydney, Australia. ${ }^{28}$ SLHD services an ethnically diverse community with $43 \%$ coming from a linguistically diverse background. ${ }^{28}$ Allied health disciplines included in the sample were physiotherapy, occupational therapy, psychology, nutrition and dietetics, speech pathology, pharmacy, podiatry, radiography, and social work. Allied health professionals who were employed in the SLHD to provide inpatient, outpatient, and community health services $(n=514)$ were invited to participate in this study.

\section{Survey tools}

The survey tool used was the validated RCCT that measures research capacity and culture across three domains: organization, team, and individual. The RCCT has a strong internal consistency and good test-retest reliability. ${ }^{18}$ Each domain contains a number of items/questions (18 questions for the organization domain, 19 questions for team domain, and 14 questions for the individual domain). Participants were asked to rate on a 1-10-point Likert-style scale if the organization, the team, or they as individuals have success or skills in the features described in each item (where $1=$ no success/skill and $10=$ high success/skill). The $\mathrm{RCCT}^{18}$ provides information 
about: 1) skills or success in research at the organization, team (or department), and individual levels; 2) barriers and motivators to performing research; 3 ) individual research activities completed in the past 12 months; 4) basic information regarding the individual's work role, qualifications obtained (ie, certificate, undergraduate, postgraduate, and $\mathrm{PhD}$ ), and enrollment in higher degree study (yes/no) or other professional development related to research.

For the purposes of this study, additional participant demographics were collected to enable further exploration of any relationship between demographic characteristics and the outcomes from the RCCT. The extra questionnaire items were age, number of years in professional service, employment status, type of workplace facility and predominant work setting, specific professional level/grading, allied health discipline, and the highest qualification obtained.

\section{Procedures and equipment}

The study was conducted in the SLHD between November 2015 and May 2016. Allied health staff were invited to participate in an online survey via an email. The email invitation included a description of the study, participant information sheet, and a link to the online survey. A hardcopy of the survey was also attached to the email invitation for staff preferring to answer and return a paper version. The survey was promoted at discipline-specific team meetings, and respective discipline heads were asked to encourage staff participation. Completion of the survey was considered as consent to participate in the study. The survey was voluntary, and confidentiality of all survey participants was maintained with coding. Study data were collected and managed using Research Electronic Data Capture (REDCap) hosted by the SLHD. ${ }^{29}$ Paper-based surveys were entered manually into the REDCap database.

\section{Statistical analysis}

Quantitative data were analyzed using IBM SPSS Statistics for Windows, version 22.0 (IBM Corporation, Armonk, NY, USA). Items that were reverse phrased in the RCCT were reverse scored and reworded before the statistical analysis was performed. Likert-style scaled items within organization, team, and individual domains were summarized using descriptive statistics of median and interquartile range (IQR). These median scores were further categorized as high (median score $\geq 7$ ), moderate (median score 4-6.99), and low (median score $<4$ ). ${ }^{30}$ A Friedman test was performed to determine whether there was a difference in success/skill levels of the median scores between organization, team, and individual domains. Post hoc Wilcoxon signed-rank tests were performed to determine where the differences occurred between these domains. An exploratory factor analysis was conducted on organization, team, and individual domains to establish if similar underlying themes emerged from the three domains. Specifically, the exploratory factor analysis was performed on a nonparametric similarity matrix calculated using Spearman correlations to identify common themes of respondents within each domain of the RCCT. An oblique rotation using direct oblimin was used as items within each domain were assumed to be related to each other. ${ }^{31}$ Factors with an eigenvalue of $>1$ were retained with a parallel analysis conducted to confirm factor retention. Cronbach's alpha scores were calculated for the items representing the factors identified within each domain of the RCCT.

Correlations were performed between some of the demographic variables, which are listed in Table 1 (ie, age, number of years employed as an allied health professional, facility, seniority, allied health discipline/department, and highest qualification obtained) and the derived factor scores within each domain to determine possible relationships of these variables with research capacity. Specifically, Spearman's rho correlations were performed between ordinal demographic variables and the derived factor scores within each domain. To establish the strength of the relationship between categorical independent demographic variables (such as allied health discipline and facility) and the derived factor scores, Kruskal-Wallis output was converted to correlation values for ease of comparison. The level of significance was set at $p<0.05$.

\section{Results}

Of the 514 allied health professionals, 278 returned surveys giving a response rate of $54 \%$. Two respondents did not complete any part of the questionnaire and have not been included in the analysis. The demographic information of the 276 participants is summarized in Table 1 and represents a combination of the demographic data from the RCCT with extra questions that were included to further characterize the sample.

Items scoring the highest at the organizational level (ie, median score 27 ) were: ensures staff career pathways are available in research; engages external partners in research; promotes clinical practice based on evidence; ensures organization planning is guided by evidence; supports peer-reviewed publication of research; supports applications for research scholarships/degrees; encourages research activities relevant to practice; and has senior managers who support research (Table 2A). Lowest scoring items in this domain (ie, median 
Table I Participants' demographic data $(\mathrm{N}=276)$

\begin{tabular}{|c|c|c|c|}
\hline Variable & n (\%) & Variable & n (\%) \\
\hline Age (years) & & Current job classification & \\
\hline$<25$ & $22(8)$ & Level I & $59(2 I)$ \\
\hline $25-29$ & $80(29)$ & Level 2 & $59(21)$ \\
\hline $30-34$ & $48(17)$ & Level 3 & $49(18)$ \\
\hline $35-39$ & $31(11)$ & Level 4 & $31(11)$ \\
\hline $40-44$ & $27(10)$ & Level 5 & $17(6)$ \\
\hline $45-49$ & $19(7)$ & Level 6 & $10(4)$ \\
\hline $50-54$ & $24(9)$ & Level 7 & $3(1)$ \\
\hline $55-60$ & $14(5)$ & Level 8 & $2(1)$ \\
\hline$>60$ & II (4) & Grade I & $14(5)$ \\
\hline \multicolumn{2}{|c|}{ Number of years employed as an $\mathrm{AH}$ professional } & Grade 2 & II (4) \\
\hline$<3$ & $56(20)$ & Grade 3 & $10(4)$ \\
\hline $3-5$ & $39(14)$ & Grade 4 & $0(0)$ \\
\hline $6-10$ & $60(22)$ & Grade 5 & $\mathrm{I}(<\mathrm{I})$ \\
\hline $11-15$ & $39(14)$ & Clinical psychologist & $5(2)$ \\
\hline $16-20$ & $24(9)$ & Senior clinical psychologist & $\mathrm{I}(<\mathrm{I})$ \\
\hline$>20$ & $58(21)$ & Principal clinical psychologist & $1(<1)$ \\
\hline \multicolumn{2}{|c|}{ Number of years employed as an $\mathrm{AH}$ professional in SLHD } & Others & $3(1)$ \\
\hline$<3$ & $97(35)$ & Department & \\
\hline $3-5$ & $47(17)$ & Physiotherapy & $84(30)$ \\
\hline $6-10$ & $51(18)$ & Occupational therapy & $52(19)$ \\
\hline $11-15$ & $31(11)$ & Speech pathology & $3(1)$ \\
\hline $16-20$ & $21(8)$ & Nutrition and dietetics & $48(17)$ \\
\hline$>20$ & $29(11)$ & Pharmacy & $35(13)$ \\
\hline Current employment status & & Radiography & II (4) \\
\hline Full-time & $225(82)$ & Psychology & $7(3)$ \\
\hline Part-time & $49(18)$ & Social work & $22(8)$ \\
\hline Casual & $2(<1)$ & Podiatry & $4(1)$ \\
\hline Facility within SLHD & & Orthotics & $0(0)$ \\
\hline RPAH & $137(50)$ & Others & $10(4)$ \\
\hline $\mathrm{CRGH}$ & $103(37)$ & Highest qualification obtained & \\
\hline Balmain hospital & II (4) & Certificate & $\mathrm{I}(<\mathrm{I})$ \\
\hline Canterbury hospital & $13(5)$ & Diploma & $8(3)$ \\
\hline Community & $12(4)$ & Bachelor degree & $144(52)$ \\
\hline Predominant area of work & & Entry level masters & II (4) \\
\hline Hospital inpatients & $161(58)$ & Postgraduate certificate & $13(5)$ \\
\hline Hospital outpatients & $46(17)$ & Postgraduate diploma & $16(6)$ \\
\hline Community & $20(7)$ & Postgraduate masters course & $57(21)$ \\
\hline Management & $15(6)$ & Masters research & $8(3)$ \\
\hline Other & $34(12)$ & $\mathrm{PhD}$ & $16(6)$ \\
\hline Seniority* & & Others & $2(<1)$ \\
\hline Junior staff & $148(54)$ & Currently enrolled in educational program? & \\
\hline \multirow[t]{2}{*}{ Senior staff } & $128(46)$ & Yes & $32(12)$ \\
\hline & & No & $244(88)$ \\
\hline
\end{tabular}

Notes: *Seniority, level 3 and above or grade 2 and above or senior clinical psychologist and above.

Abbreviations: AH, Allied health; CRGH, Concord Repatriation General Hospital; RPAH, Royal Prince Alfred Hospital; SLHD, Sydney Local Health District.

score of $\leq 5$ ) were: has funds, equipment, or administration to support research activities; has software programs for analyzing research data; and has mechanisms to monitor research quality (Table 2A). Similarly, the highest scoring items at the team level (ie, median score of $\geq 7$ ) were: disseminates research results at research forums/seminars; does planning that is guided by evidence; has team leaders who support research; and conducts research activities relevant to practice (Table
2B). The lowest scoring items at the team level (ie, median score of $\leq 4$ ) were: has funds, equipment, or administration to support research activities; and has software to support research activities (Table 2B). At the individual level, items that scored highest (ie, median score of $\geq 7$ ) were: critically reviewing the literature and finding relevant literature (Table $2 \mathrm{C})$. Ten of the 19 items at the individual level had a median score of $\leq 5$, and the lowest scoring items (ie, median score 
Table 2 Organization, team, and individual success/skill levels $(\mathrm{N}=265)$

\begin{tabular}{|c|c|c|c|c|c|c|}
\hline \multicolumn{7}{|c|}{ (A) Organizational success or skill level $(n=265)$} \\
\hline Item $^{a}$ & Description & $\begin{array}{l}\text { Research } \\
\text { infrastructure }^{b} \\
\text { (factor loading) }\end{array}$ & $\begin{array}{l}\text { Research } \\
\text { culture } \\
\text { (factor } \\
\text { loading) } \\
\end{array}$ & Median $^{d}$ & IQR $^{\mathrm{d}}$ & $\begin{array}{l}\% \\
\text { Unsure }\end{array}$ \\
\hline ii & Has funds, equipment, or admin to support research activities ${ }^{b}$ & 0.97 & & 5 & $3-7$ & 16 \\
\hline i & Has adequate resources to support staff research training ${ }^{\mathrm{b}}$ & 0.93 & & 6 & $3-8$ & 12 \\
\hline$x i$ & Has software programs for analyzing research data ${ }^{\mathrm{b}}$ & 0.92 & & 5 & $2-7$ & 30 \\
\hline xii & Has mechanisms to monitor research quality ${ }^{b}$ & 0.83 & & 5 & $3-7$ & 29 \\
\hline iii & Has a plan or policy for research development ${ }^{b}$ & 0.74 & & 6 & $4-8$ & 26 \\
\hline viii & Accesses external funding for research ${ }^{\mathrm{b}}$ & 0.71 & & 6 & $3-7$ & 27 \\
\hline v & Ensures staff career pathways are available in research ${ }^{\mathrm{b}}$ & 0.66 & & 7 & $3-7$ & 15 \\
\hline xiv & Supports a multidisciplinary approach to research ${ }^{\mathrm{b}}$ & 0.64 & & 6 & $4-8$ & 11 \\
\hline$x v i$ & Engages external partners (eg, universities) in research ${ }^{\mathrm{b}}$ & 0.64 & & 7 & $4-8$ & 14 \\
\hline xiii & Has identified experts accessible for research advice ${ }^{b}$ & 0.52 & & 6 & $5-8$ & 18 \\
\hline vii & Has consumers involved in research ${ }^{\mathrm{b}}$ & 0.49 & & 6 & $4-7$ & 19 \\
\hline ix & Promotes clinical practice based on evidence ${ }^{c}$ & & 1.00 & 8 & $7-9$ & 5 \\
\hline vi & Ensures organization planning is guided by evidence ${ }^{c}$ & & 0.80 & 7 & $5-8$ & 13 \\
\hline xviii & Supports the peer-reviewed publication of research ${ }^{c}$ & & 0.69 & 7 & $5-8$ & 19 \\
\hline xvii & Supports applications for research scholarships/degrees ${ }^{c}$ & & 0.64 & 7 & $4-8$ & 19 \\
\hline$x$ & Encourages research activities relevant to practice ${ }^{c}$ & & 0.62 & 7 & $5-8$ & 9 \\
\hline iv & Has senior managers who support research ${ }^{c}$ & & 0.58 & 7 & $5-9$ & 9 \\
\hline xv & Has regular forums/bulletins to present research findings ${ }^{c}$ & & 0.51 & 6 & $4-8$ & 12 \\
\hline \multicolumn{7}{|c|}{ (B) Team success or skill level $(n=250)$} \\
\hline Item $^{\text {e }}$ & Description & $\begin{array}{l}\text { Research } \\
\text { orientation }^{\mathrm{f}} \\
\text { (factor loading) }\end{array}$ & $\begin{array}{l}\text { Research } \\
\text { supportg } \\
\text { (factor } \\
\text { loading) } \\
\end{array}$ & Median $^{d}$ & IQR $^{\mathrm{d}}$ & $\begin{array}{l}\% \\
\text { Unsure }\end{array}$ \\
\hline xiv & Disseminates research results at research forums $/$ seminars $^{f}$ & 0.98 & & 7 & $4-9$ & 11 \\
\hline xi & Supports applications for research scholarships/degrees ${ }^{f}$ & 0.91 & & 6 & $4-8$ & 16 \\
\hline vii & Does planning that is guided by evidence ${ }^{f}$ & 0.87 & & 7 & $5-8$ & 7 \\
\hline xviii & Supports peer-reviewed publication of research ${ }^{f}$ & 0.85 & & 6 & $4-8$ & 13 \\
\hline xiii & Has identified experts accessible for research advice ${ }^{f}$ & 0.84 & & 6 & $4-8$ & 13 \\
\hline $\mathrm{v}$ & Has team leaders who support research ${ }^{f}$ & 0.82 & & 7 & $5-8$ & 5 \\
\hline$x v$ & Supports a multidisciplinary approach to research ${ }^{f}$ & 0.76 & & 6 & $4-8$ & 10 \\
\hline xvii & Has external partners (eg, universities) engaged in research ${ }^{f}$ & 0.76 & & 5 & $3-8$ & 16 \\
\hline$x$ & Conducts research activities relevant to practice ${ }^{f}$ & 0.74 & & 7 & $4-8$ & 7 \\
\hline xii & Has mechanisms to monitor research quality ${ }^{f}$ & 0.72 & & 6 & $4-8$ & 21 \\
\hline ix & Has applied for external funding for research ${ }^{f}$ & 0.53 & & 5 & $3-7$ & 23 \\
\hline vi & Provides opportunities to get involved in research fig & 0.50 & 0.45 & 6 & $4-8$ & 5 \\
\hline ii & Has funds, equipment, or admin to support research activities ${ }^{g}$ & & 1.00 & 4 & $2-6$ & 13 \\
\hline i & Has adequate resources to support staff research training ${ }^{g}$ & & 0.89 & 5 & $3-7$ & 11 \\
\hline iii & Does team-level planning for research development ${ }^{g}$ & & 0.81 & 5 & $3-7$ & 10 \\
\hline iv & Ensures staff involvement in developing that plan ${ }^{g}$ & & 0.69 & 5 & $3-7$ & 8 \\
\hline xix & Has software available to support research activities ${ }^{g}$ & & 0.68 & 4 & $2-7$ & 25 \\
\hline viii & Has consumer involvement in research activities/planning ${ }^{g}$ & & 0.67 & 5 & $3-7$ & 17 \\
\hline $\mathrm{xvi}$ & Has incentives and support for mentoring activities ${ }^{g}$ & & 0.52 & 5 & $3-7$ & 12 \\
\hline \multicolumn{7}{|c|}{ (C) Individual skill level $(n=250)$} \\
\hline Item $^{\text {h }}$ & Description & $\begin{array}{l}\text { Research skill of } \\
\text { the individual' } \\
\text { (factor loading) }\end{array}$ & Median $^{\mathrm{d}}$ & IQR $^{\mathrm{d}}$ & \% Unsure & \\
\hline iv & Writing a research protocoli & 0.90 & 5 & $3-7$ & 3 & \\
\hline xii & Writing a research report $\mathrm{t}^{\mathrm{i}}$ & 0.89 & 5 & $3-7$ & 1 & \\
\hline xiii & Writing for publication in peer-reviewed journals' & 0.86 & 4 & $2-7$ & 3 & \\
\hline xiv & Providing advice to less experienced researchers ${ }^{i}$ & 0.83 & 3 & $1-6$ & 2 & \\
\hline ix & Using computer data management systems ${ }^{i}$ & 0.82 & 5 & $2-7$ & 3 & \\
\hline
\end{tabular}


Table 2 (Continued)

\begin{tabular}{|c|c|c|c|c|c|}
\hline \multicolumn{6}{|c|}{ (C) Individual skill level $(n=250)$} \\
\hline Item $^{\mathrm{h}}$ & Description & $\begin{array}{l}\text { Research skill of } \\
\text { the individual' } \\
\text { (factor loading) }\end{array}$ & Median $^{\text {d }}$ & IQR $^{\mathrm{d}}$ & $\%$ Unsure \\
\hline$\overline{x i}$ & Analyzing quantitative research data & 0.82 & 5 & $2-7$ & I \\
\hline vi & Submitting an ethics application ${ }^{\mathrm{i}}$ & 0.82 & 3 & $1-6$ & 4 \\
\hline viii & Collecting data, eg, surveys and interviewsi & 0.79 & 6 & $5-8$ & I \\
\hline$x$ & Analyzing qualitative research data & 0.79 & 5 & $2-7$ & $<1$ \\
\hline ii & Critically reviewing the literature $\mathrm{i}^{\mathrm{i}}$ & 0.75 & 7 & $6-8$ & $<1$ \\
\hline vii & Designing questionnaires ${ }^{i}$ & 0.75 & 5 & $3-7$ & 2 \\
\hline $\mathrm{i}$ & Finding relevant literature $\mathrm{e}^{\mathrm{i}}$ & 0.72 & 7 & $6-8$ & $<1$ \\
\hline iii & Using a computer referencing system (eg, Endnote) ${ }^{i}$ & 0.67 & 6 & $3-8$ & I \\
\hline$v$ & Securing research funding' & 0.65 & 2 & $1-4$ & 4 \\
\hline
\end{tabular}

Notes: altem numbers i-xviii represent the original sequential order of questions in the RCCT. ${ }^{b}$ Research infrastructure available in the organization. ${ }^{\mathrm{C}}$ Research culture within the organization. ${ }^{d}$ Success/skill level on a $I-10$ scale ( $I=$ no success/skill and $10=$ highest possible success/skill). ${ }^{\text {eltem numbers } i-x i x}$ represent the original sequential order of questions in the RCCT. 'Research orientation within the team. ${ }^{8}$ Research support within the team. ${ }^{\text {III }}$ tem numbers $\mathrm{i}-\mathrm{xiv}$ represent the original sequential order of questions in the RCCT. Individual skill level in carrying out the research.

Abbreviations: IQR, interquartile range; RCCT, Research Capacity in Context Tool.

4) were: writing for publication in peer-reviewed journals; providing advice to less experienced researchers; submitting an ethics application; and securing research funding (Table 2C). Individual allied health professional research activities undertaken in the past 12 months were: collected data via surveys and interviews $(66 \%, \mathrm{n}=165)$; analyzed quantitative research data $(41 \%, \mathrm{n}=103)$; and written a research report or presentation ( $39 \%, \mathrm{n}=98$; Table S1A).

There was a significant difference in the overall median scores for the organization, team, and individual domains of the RCCT (Friedman $\chi^{2}(2)=42.04, p<0.001$ ). Post hoc analysis with Wilcoxon signed-rank tests demonstrated that the organization domain had a significantly higher median (IQR) score of $6(5-8)$ than the team domain $6(4-8)(Z=-3.53$, $p<0.001)$ and that both the organization and team domains' median scores were significantly higher than that of the individual domain, 5 (3-7) (organization versus individual domain $Z=-5.83, p<0.001$; team versus individual domain $Z=-4.23, p<0.001$; Table 2).

Approximately $32 \%(n=80)$ of participants reported that research-related activities were part of their role (Table S1B). The support available to individual allied health professionals to conduct research was reported to be highest for library access $(57 \%, n=46)$. For those allied health professionals who identified that research was part of their role, approximately $40 \%$ or less reported that they had adequate research support in terms of computer software, research supervision, time, research funds, administrative support, and training (Table S1B). The main barriers to research reported by participants included: lack of time for research $(91 \%$, $\mathrm{n}=228)$; other work roles taking priority $(83 \%, \mathrm{n}=208)$; lack of suitable backfill requiring a staff member to perform extra duties for employees on vacation or assigned to other work roles $(50 \%, \mathrm{n}=125)$; a desire for work/life balance $(49 \%$, $n=123)$; and lack of skills for research $(49 \%, n=123$; Table $\mathrm{S} 2 \mathrm{~A})$. The main motivators to performing research reported by participants included: to develop skills $(84 \%, n=210)$; to increase job satisfaction $(65 \%, \mathrm{n}=163)$; for career advancement $(56 \%, \mathrm{n}=140)$; to keep the brain stimulated $(50 \%$, $\mathrm{n}=125)$; or identifying a problem that needed changing $(49 \%$, $\mathrm{n}=123$; Table S2B).

The data were suitable for exploratory factor analysis as the sample was large enough, with greater than the recommended 10 cases for each variable to be factor analyzed with correlation coefficients for items within each domain $>0.3^{32}$ (Table 3). The Kaiser-Meyer-Olkin value for each of the domains assessed was greater than the recommended value of $0.6^{32}$ with Bartlett's test of sphericity $(p<0.001)$ supporting the factorability of the respective correlation matrices (Table 3). Inspection of the scree plots and eigenvalues, along with a parallel analysis, confirmed the retention of two factors for both the organization and team domains, and one factor for the individual domain of the RCCT (Table 3) as per the generated 95th percentile eigenvalues. As all items within each domain demonstrated a strong correlation $(>0.3)$ against one another in the component correlation matrix (Table 3 ), exploratory factor analyses were conducted separately for the organization, team, and individual domains of the RCCT using an oblique direct oblimin rotation. ${ }^{32}$

Exploratory factor analysis resulted in the identification of two key factors for the organization domain based on items that clustered around these factors (Table 2). For the 


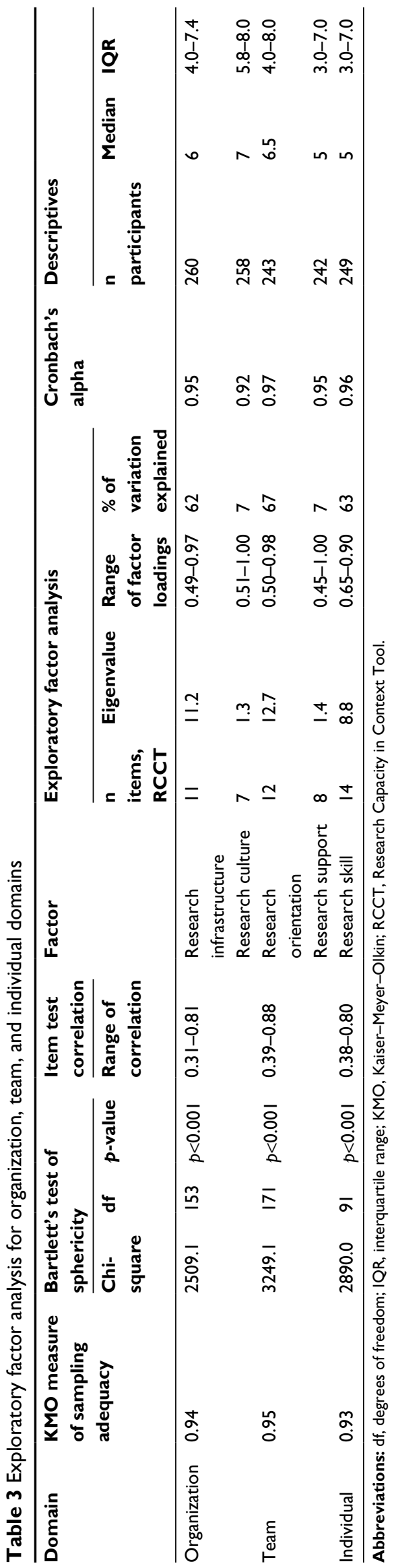

organization domain, one factor represented the research infrastructure available within the organization (11 items: items i-iii, v, vii-viii, xi-xiv, xvi of the organization domain) with factor loading range 0.49-0.97 (Tables 2 and 3), and the second factor represented the research culture within the organization (seven items: items iv, vi, ix-x, $\mathrm{xv}$, xvii-xviii in the organization domain) with factor loading range $0.51-1.0$ (Tables 2 and 3). These two factors had eigenvalues of 11.2 and 1.3 , respectively, and in combination explained $69 \%$ of the variation of the participants' perception of the organization's success/skill level in relation to research (Table 3). Median (IQR) scores for the factors of research infrastructure and research culture within the organization were $6(4.0-7.4)$ and 7 (5.8-8.0), respectively (Tables 2 and 3). Wilcoxon signed-rank tests between these two factors demonstrated that the research culture within the organization had a significantly higher median (IQR) score than the research infrastructure available within the organization $(Z=-10.47, p<0.001$; Tables 2 and 3$)$.

Similarly, two factors were identified within the team domain, and items that clustered around these suggested that the first factor was the research orientation within the team (12 items: items v-vii, ix-xi, xii-xv, xvii-xviii on the team domain) with factor loading range $0.50-0.98$ (Tables 2 and 3), and the second factor was the research support within the team (eight items: items i-iv, vi, viii, xvi, xix on the team domain) with factor loading range $0.45-1.00$ (Tables 2 and 3). These two factors had eigenvalues of 12.7 and 1.4 , respectively, and in combination explained $74 \%$ of the variation of the participants' perception of the team's current success/skill level in relation to research (Table 3). Median (IQR) scores for research orientation and research support within the team were $6.5(4.0-8.0)$ and 5 (3.0-7.0), respectively (Tables 2 and 3). Wilcoxon signed-rank tests between these two factors demonstrated that the research orientation within the team had a significantly higher median score than the research support within the team $(Z=-9.64$, $p<0.001$; Tables 2 and 3).

Only one factor was identified within the individual domain, and items that clustered around this suggested that this factor was the research skill of the individual (14 items: items $\mathrm{i}-\mathrm{xiv}$ on the individual domain) with factor loading range $0.65-0.90$ (Tables 2 and 3). This factor had an eigenvalue of 8.8 and explained $63 \%$ of the variation of participants' perception of their own current success/skill level in relation to research with a median (IQR) value of 5 (3.0-7.0) (Table 3).

The Cronbach's alpha scores for each of the abovementioned factors demonstrated values of $>0.8$ indicating very 
good internal reliability ${ }^{32}$ of items clustering around factors identified for each domain of the RCCT (organization, team, and individual domains; Table 3).

The strength of relationships between demographic variables and factors identified within various domains of the RCCT was examined (Table 4). There was a small to medium effect size observed between the department in which the participants worked and each of the factors identified within each domain. A small to medium effect size was also observed between the participants' highest qualifications and the factor research orientation in the team domain, and the factor research skill in the individual domain (Table 4).

\section{Discussion}

This study contributes to the understanding of key factors influencing the research capacity of the allied health workforce within a large Australian metropolitan health district. The response rate in this study was $54 \%$ which was in the upper range of the response rates reported by other studies (13-64\%). ${ }^{3,18,20-23,25,26,30}$ The main findings were that research capacity and culture within the organization and team domains were rated more highly by participants in terms of success/skill levels than in the individual domain. In addition, two key factors were identified that impacted research within each of the organization and team domains. In the organization domain, these factors were the research infrastructure available within the organization and the research culture within the organization. In the team domain, the factors were the research orientation and research support within the team. In the individual domain, only one key factor, the research skill level of the individual was identified as impacting research capacity. These factors were different in each domain suggesting that, to build research capacity and culture, different strategies would be required within each of these domains.

Similar to other studies, ${ }^{6,7,12,18,23}$ our study found that research capacity and culture at the organization and team domains were reported to be higher than at the individual domain (Table 2). Participants rated the overall organization and team domains (median [IQR] 6 [5-8] and 6 [4-8], respectively) as being at the upper end of moderately successful for research capacity, whereas the ratings for the individual domain (median [IQR] 5 [3-7]) were at the lower end of moderately successful. Most individuals reported a lack of involvement in research because of a lack of skills and confidence in carrying out research, particularly in items such as writing for publication in peer-reviewed journals, providing advice to less experienced researchers, submitting ethics applications, and securing research funding (Table 2C). Low levels of research confidence in the clinical setting g $^{6,27,33}$ (Tables 2C and S1) may be due to the lack of research training and mentoring in the workplace, ${ }^{8,27,33}$ as allied health professionals receive only basic research training in their undergraduate professional training programs.

Interestingly, participants' "unsure" responses to the survey items within each domain were as high as $30 \%$ in the organization domain, and $25 \%$ in the team domain, but less than $5 \%$ in the individual domain (Table 2). Similar findings have been reported in a study utilizing the RCCT in community health services, ${ }^{30}$ where "unsure" responses were $59 \%$ in the organization domain, $52 \%$ in the team domain, but only $10 \%$ in individual domain. The high percentage of "unsure" responses in the organization and team domains may indicate that participants were unaware of how their organization or team was performing in terms of research culture and support for research, whereas the low percentage of "unsure" responses in the individual domain indicated that individuals were aware of their own research skill levels. Strategies may be required to increase allied health staff awareness of the facilities and support available within these domains which may assist in encouraging individual research.

\section{Key factors affecting research capacity Organization domain factors and implications}

The two factors impacting the organization domain were the research infrastructure available and the research culture

Table 4 Strength of relationship between significant demographic variables and factors identified

\begin{tabular}{lllll}
\hline Domain & Factor & Demographic & Correlation & $p$-value \\
\hline Organization & Research infrastructure & Department & $r=0.28$ & $p<0.0$ I \\
& Research culture & Department & $r=0.20$ & $p<0.05$ \\
Team & Research orientation & Department & $r=0.34$ & $p<0.0$ I \\
& & Highest qualification & $r_{s}=0.20$ & $p<0.05$ \\
& Research support & Department & $r=0.25$ & $p<0.0$ I \\
Individual & Research skill & Department & $r=0.26$ & $p<0.0$ I \\
& & Highest qualification & $r_{s}=0.36$ & $p<0.01$ \\
\hline
\end{tabular}

Notes: $r$, Spearman's rho correlation; $r$, effect size correlation for Kruskal-Wallis test. 
within the organization. The organization was rated by participants as having a high research culture (median [IQR] 7 [5.8-8.0]; Table 3). A positive research culture has been described as an organization where research is valued and is undertaken by the majority of clinicians and includes the implementation of evidence-based findings in clinical practice. ${ }^{34}$ Such a culture is believed to influence the adoption of research initiatives and behaviors of individual allied health professionals employed within the organization. ${ }^{12,30,35}$ Ensuring that research is explicitly cited in the strategic plan and mission statements of the organization ${ }^{12,36-38}$ is a strategy that may foster a research culture by demonstrating that research is highly valued and integral to the work of the organization. The other key factor identified in the organization domain was research infrastructure available to support research. Infrastructure, at the level of the organization, was rated by participants in this study as moderate (median [IQR] 6 [4.0-7.4]; Table 3) which most likely reflects the resources available within the health district to support research (eg, two ethics committees, a close association with medical research institutes and a large research intensive university, and strategic plans that incorporate research as a key performance indicator across the organization ${ }^{39,40}$ ). Structures and processes that are research enablers include ethics committees, grant schemes, collaborations with external partners, academic affiliations, or research institutes. ${ }^{1,8}$ Grant schemes and collaborations have been shown to facilitate mentoring which leads to enhanced research skills and confidence in novice researchers. ${ }^{33,41}$ Other infrastructure provided by an organization that supports research in the clinical setting includes access to libraries and library resources such as databases for literature searches, access to computers with appropriate software such as statistical programs, appropriate workspaces to conduct research, and administrative support to assist clinician researchers. ${ }^{13,30,42}$

\section{Team domain factors and implications}

Two key factors impacting research at the team level included the research orientation and research support within the team. Research orientation was rated at the upper end of moderately successful (median [IQR] 6.5 [4.0-8.0]). The concept of research orientation encompasses several different components: the attitude toward research and the overall value placed on research, involvement in research (eg, research execution and output), evidence-based practice, being at the leading edge of the profession by integrating research findings into clinical practice, and keeping up to date with new knowledge and information. ${ }^{43,44}$ Research orientation within the team domain includes the sharing of knowledge and research expertise within a project, which may assist in building research skills in novice researchers, thereby increasing overall research activities. ${ }^{841}$ The other factor, research support within the team, was rated as moderately successful (median [IQR] 5 [3.0-7.0]). Research support offered within the team also includes library resources such as databases, access to computers and software, workspaces to conduct research and data collection, funds, and equipment and administrative support. ${ }^{13,30,42}$

One strategy that may promote research orientation and research support at the team level is to link novice and experienced researchers (eg, university academics and other key researchers within an organization) within a designated research team, as this approach may assist the transfer of knowledge, thereby enhancing the novice's ability to do research. ${ }^{8}$ Linking academics with novice clinician researchers will help to ensure that the research has clinical relevance while also assisting novice clinician researchers to complete the full research cycle (ie, research project design and implementation, and data collection and analysis, with appropriate dissemination of results via conference presentations and publications). ${ }^{41}$

Additional strategies that have been suggested as important enablers to the success and performance of team-based research activities that may promote research orientation and research support include flexible work arrangements to ensure protected time for carrying out research, ${ }^{1,8,41}$ designated research roles within allied health departments, ${ }^{35,45,46}$ and grant funding. ${ }^{33}$ Designated research positions and making research a part of job descriptions within allied health departments have been shown to enhance the culture of research and evidence-based practices across an organization and team with increases in research activity and research outputs (ie, journal publications and conference presentations). ${ }^{6,8,12,23,35,46}$

Research registers within clinical departments or research teams, while capturing and monitoring research activity, may also motivate research teams through greater recognition of research output. ${ }^{2,47}$ Registers should include both traditional measures (eg, details of successful grant submissions, publications, and presentations) and nontraditional measures (eg, increased confidence levels in performing research, attaining methodological skills, and greater interest in conducting research) of research performance..$^{2,33,45}$

\section{Individual domain factors and implications}

At the individual level, only one key factor was identified as impacting research capacity which was the research skill of 
the individual. Items within this factor that were rated highest in terms of success/skill levels (median score of 7) were: finding relevant literature and critically reviewing the literature (Table 2C). These items relate to evidence-based practice and demonstrate that individuals feel that they possess the skills involved in sourcing and critically appraising the literature to guide practice in the clinical setting. The remaining items within this factor (items iii-xiv) were related to the process and mechanics of carrying out research and were scored by individuals as low to moderate (median scores 2-6) with the lowest success/skill levels (ie, median score $\leq 4$ ) reported as securing research funding, submitting an ethics application, writing for publication in a peer-reviewed journal, and providing advice to less experienced researchers (Table 2C). This indicates that the individual skills required to carry out research are limited and this finding is reflected in the low numbers of individuals in our study undertaking research activities within the last 12 months (Table S1A and B). This is similar to previous studies that found that individuals scored more highly in undertaking research activities related to evidence-based practice (ie, sourcing and critically reviewing the literature), with low skill levels in the key elements of carrying out research. ${ }^{6,17,18}$

Educational strategies that build research skills need to be tailored to individual needs and interests to be effective. ${ }^{27,33}$ Our study highlighted that participants' success/skill ratings for the majority of items in the individual domain (ie, items iii-xiv) scored low to moderate, highlighting that educational initiatives should target the key elements of carrying out research with particular emphasis on writing a research protocol, writing a research report, writing for publication in peer-reviewed journals, using computer data management systems, analyzing quantitative and qualitative research data, submitting ethics applications, designing questionnaires, and securing research funding (eg, grant writing; Table 2C). Allied health professionals will often report low individual skill levels in research which leads to a perception that individuals do not have the ability to undertake research. ${ }^{19-22,48-50}$ Educational strategies, along with mentoring through involvement in team-based research, may increase confidence and skill levels that enable allied health professionals to undertake research.

\section{Motivators and barriers to allied health research}

The majority of allied health participants in this study reported that the main motivators to be involved in research were to develop skills, to increase job satisfaction, and to keep the brain stimulated, an identified problem that needs changing (Table S2B). This is consistent with previous studies that have shown that an interest in research, viewing research as important to being a professional, and a desire to improve clinical practice are attitudes that may facilitate involvement of individuals in research. ${ }^{4,48,51,52}$ Strategies to build research capacity should initially target individuals with an interest in research who self-select to the role of clinician researcher. ${ }^{2,7,52}$ These individuals should also be supported by the organization and through mentoring as such support has been identified as important in facilitating research outcomes even in highly motivated clinicians. ${ }^{4}$

The most important barriers to research reported by allied health participants were lack of time, other work roles taking priority, lack of suitable backfill, lack of skills for research, and lack of funds for research (Table S2A). These results were similar to those reported by other studies with the majority of studies citing lack of time and motivation, lack of backfill arrangements and funding, coupled with lack of support from managers and colleagues to facilitate research in the clinical setting, as factors affecting the research capacity of individuals. ${ }^{1-5,7,12,13,33,41,53,54}$ Strategies such as dedicated research positions, ${ }^{6,41,46}$ targeted education toward individual and team needs, ${ }^{8,41}$ mentoring, ${ }^{27,33,54,55}$ and funding for appropriate backfill of positions ${ }^{1,5,8}$ have been suggested as strategies for addressing some of these barriers in an effort to assist those with an interest in research to develop their individual research skills.

\section{Demographic variables potentially affecting research capacity}

Our study identified two other variables that may impact research capacity and culture at the individual level. These were the department in which the individual worked and the level of qualification. A small to medium effect size between the allied health department in which the participants worked and the various factors identified within each domain of the RCCT (Table 4) suggested that there may be an association between the department in which the participants worked and research capacity. In Australia, some allied health professional training degrees have research projects linked to the attainment of their professional qualifications. Many of these projects occur in the clinical departments within the related disciplines which creates a positive research environment with clinician researchers supporting and mentoring students' research projects.

The level of qualification attained by an individual also may affect research capacity. Our study showed a small to medium positive effect size between the participants' highest 
qualification and the key factors of research orientation in the team domain, and research skill in the individual domain, suggesting that higher qualifications may influence research capacity (Table 4). Such findings have been reported in a previous study which showed that the highest qualification obtained was a significant predictor of involvement in research. ${ }^{20}$ Higher degree qualifications (ie, research masters and doctoral degrees) are research intensive and provide individuals with the necessary skills to perform clinical research and to support team members in clinical research. In our study only a small percentage of participants had completed a masters by research degree $(3 \%, \mathrm{n}=8)$, or a $\mathrm{PhD}$ $(6 \%, n=16)$ with $21 \%$ of participants, $(n=57)$ reporting that they had completed a postgraduate masters course (Table 1). The low numbers of participants with research degrees in our study may help to explain the low to moderate success/skill ratings for items relating to individual research skills (Table 2C). Our study did not show any significant relationships between age, recency of practice, seniority, and current job classification with research capacity which is contrary to the findings reported previously by some studies..$^{6,12,20,23}$

\section{Whole system approach to research capacity building}

The key factors identified within the organization, team, and individual domains, highlight the importance of a "whole system approach" to allied health research capacity building. ${ }^{1,6,13,17,27}$ It is important to recognize that factors at the level of the organization will impact research capacity at the team level which will in turn impact research capacity at the individual level. Organization and team factors that support research have been identified as having a large influence on individual health professionals' skills and confidence in planning and engaging in clinical research. ${ }^{1,33,41,53}$ It is possible that infrastructure within an organization will also assist research teams throughout all phases of the research cycle, thereby ensuring successful completion of research projects. ${ }^{41,42}$ It is also important to add that there is a paucity of literature regarding the strategies that can be implemented at the organization, team, and individual levels to promote research capacity building. Many studies have offered suggestions as to interventions that may assist research capacity building at these different levels but few studies exist on the effectiveness of these interventions. ${ }^{17,41,54}$ Studies have tended to focus on team-based and individuallevel interventions within health care institutions and at present there are no randomized controlled trials investigating any of the interventions aimed at building research capacity with little evidence supporting the effectiveness of some interventions over others. ${ }^{17,41,45-47,54}$

\section{Limitations of the study}

There were a number of limitations of this study. Although the overall response rate to the RCCT was good, this study was conducted in one large metropolitan health district which limits the overall generalizability of the results to other health districts. Participant bias may have occurred in this study as those interested in research may have preferentially responded to the RCCT, thus overinflating the level of research interest. However, as all allied health staff were encouraged to participate, this is less likely. The study population was allied health professionals employed in the public health system and may not reflect the attitudes and opinions of those employed in the private health sector. As the RCCT does not adequately define what is meant by an organization or team, respondents to the survey may have interpreted these terms differently which may have affected the responses.

\section{Conclusion}

The novel finding of our study was the identification of key factors in the organization, team, and individual domains of the RCCT that impacted research capacity and culture in a large Australian metropolitan health district. The factors were the research infrastructure available and the research culture at the level of the organization, the research orientation and research support offered at the team level, and the individual's research skills at the individual level. These key factors indicate that to build research capacity for allied health professionals working in a large metropolitan health district strategies need to address each of these factors. Importantly, future research is needed to determine which strategies are the most effective at building research capacity, and in which domains these strategies should be implemented to achieve the best possible outcomes.

\section{Acknowledgments}

We would like to acknowledge the help provided in an initial consultation with the developers of the RCCT. This study used the REDCap data management system which is supported by NIH/NCRR Colorado CTSI grant number UL1 RR025780. The contents of the study are the authors' sole responsibility and do not necessarily represent official National Institutes of Health views.

\section{Disclosure}

The authors report no conflicts of interest in this work. 


\section{References}

1. Pickstone $\mathrm{C}$, Nancarrow $\mathrm{S}$, Cooke $\mathrm{J}$, et al. Building research capacity in the allied health professions. Evid Policy. 2008;4(1):53-68.

2. Skinner EH, Williams CM, Haines TP. Embedding research culture and productivity in hospital physiotherapy departments: challenges and opportunities. Aust Health Rev. 2015;39(3):312-314.

3. Paget SP, Lilischkis KJ, Morrow AM, Caldwell PHY. Embedding research in clinical practice: differences in attitudes to research participation among clinicians in a tertiary teaching hospital. Intern Med J. 2014;44(1):86-89.

4. Harvey D, Plummer D, Nielsen I, Adams R, Pain T. Becoming a clinician researcher in allied health. Aust Health Rev. 2016;40(5):562.

5. Skinner EH, Hough J, Wang YT, et al. Physiotherapy departments in Australian tertiary hospitals regularly participate in and disseminate research results despite a lack of allocated staff: a prospective crosssectional survey. Physiother Theory Pract. 2015;31(3):200-206.

6. Williams C, Miyazaki K, Borkowski D, McLinstry C, Cotchet M, Haines T. Research capacity and culture of the Victorian public health allied health workforce is influenced by key research support staff and location. Aust Health Rev. 2015;39(3):303-311.

7. Pager S, Holden L, Golenko X. Motivators, enablers, and barriers to building allied health research capacity. J Multidiscip Healthc. 2012;5:53-59.

8. Cooke J. A framework to evaluate research capacity building in health care. BMC Fam Pract. 2005;6:44.

9. Bateman H, Walter F, Elliot J. What happens next? Evaluation of a scheme to support primary care practitioners with a fledgling interest in research. Fam Pract. 2004;21(1):83-86.

10. van Weel C, Rosser WW. Improving health care globally: a critical review of the necessity of family medicine research and recommendations to build research capacity. Ann Fam Med. 2004;2(suppl 2):S5-S16.

11. Wilkes L, Cummings J, McKay N. Developing a culture to facilitate research capacity building for clinical nurse consultants in generalist paediatric practice. Nurs Res Pract. 2013;2013:709025.

12. Borkowski D, McKinstry C, Cotchett M, Williams C, Haines T. Research culture in allied health: a systematic review. Aust J Prim Health. 2016;22(4):294-303.

13. Golenko X, Pager S, Holden L. A thematic analysis of the role of the organisation in building allied health research capacity: a senior managers' perspective. BMC Health Serv Res. 2012;12:276.

14. Brody AS, Donnelly LF. Building a culture of research among clinical pediatric radiologists: a multifaceted, programmatic approach. Pediatr Radiol. 2009;39(4):367-370.

15. Farmer E, Weston K. A conceptual model for capacity building in Australian primary health care research. Aust Fam Physician. 2002;31(12):1139-1142.

16. Thomas $\mathrm{P}$, While A; West London Research Network. Increasing research capacity and changing the culture of primary care towards reflective inquiring practice: the experience of the West London Research Network (WeLReN). J Interprof Care. 2001;15(2):133-139.

17. Holden L, Pager S, Golenko X, Ware RS, Weare R. Evaluating a teambased approach to research capacity building using a matched-pairs study design. BMC Fam Pract. 2012;13:16.

18. Holden L, Pager S, Golenko X, Ware RS. Validation of the research capacity and culture (RCC) tool: measuring RCC at individual, team and organisation levels. Aust J Prim Health. 2012;18(1):62-67.

19. Finch E, Cornwell P, Nalder E, Ward E. Uncovering motivators and stumbling blocks: exploring the clinical research experiences of speechlanguage pathologists. Int J Speech Lang Pathol. 2015;17(2):138-147.

20. Finch E, Cornwell P, Ward EC, McPhail SM. Factors influencing research engagement: research interest, confidence and experience in an Australian speech-language pathology workforce. BMC Health Serv Res. 2013;13(1):144.

21. Pighills AC, Plummer D, Harvey D, Pain T. Positioning occupational therapy as a discipline on the research continuum: results of a cross-sectional survey of research experience. Aust Occup Ther J. 2013;60(4):241-251.
22. Harvey D, Plummer D, Pighills A, Pain T. Practitioner research capacity: a survey of social workers in Northern Queensland. Aust Soc Work. 2013;66(4):540-554.

23. Howard AJ, Ferguson M, Wilkinson P, Campbell KL. Involvement in research activities and factors influencing research capacity among dietitians. J Hum Nutr Diet. 2013;26(suppl 1):S180-S187.

24. Williams C, Lazzarini P. Understanding the research capacity and culture of Australian podiatrists. J Foot Ankle Res. 2015;8(suppl):2.

25. Lazzarini PA, Geraghty J, Kinnear EM, Butterworth M, Ward D. Research capacity and culture in podiatry: early observations within Queensland Health. J Foot Ankle Res. 2013;6(1):1.

26. Agustin C, Grand M, Gebski V, Turner S. Radiation therapists' perspective on barriers to clinical trials research. JMed Imaging Radiat Oncol. 2008;52(2):178-182.

27. Ried K, Farmer EA, Weston KM. Setting directions for capacity building in primary health care: a survey of a research network. BMC Fam Pract. 2006;7:8.

28. Sydney Local Health District. A Picture of Health - Sydney Local Health District; 2015:1-61. Available from: https://www.slhd.nsw.gov. au/planning/pdf/SLHD_Health_Profile.pdf. Accessed Dec 5, 2016.

29. Harris PA, Taylor R, Thielke R, Payne J, Gonzalez N, Conde JG. Research electronic data capture (REDCap) - a metadata-driven methodology and workflow process for providing translational research informatics support. J Biomed Inform. 2009;42(2):377-381.

30. Friesen EL, Comino EJ. Research culture and capacity in community health services: results of a structured survey of staff. Aust J Prim Health. 2017;23(2):123-131.

31. Field AP. Discovering Statistics Using SPSS. 3rd ed. London: SAGE; 2009.

32. Pallant JF. SPSS Survival Manual: A Step by Step Guide to Data Analysis Using IBM SPSS. 6th ed. Sydney: Allen \& Unwin; 2016.

33. Ried K, Farmer EA, Weston KM. Bursaries, writing grants and fellowships: a strategy to develop research capacity in primary health care. BMC Fam Pract. 2007;8:19.

34. Thomas V. "Think research" in everyday clinical practice: fostering research culture in health care settings. Oman Med J. 2011;26(2): 75-76.

35. Perry LIN, Grange A, Heyman BOB, Noble P. Stakeholders' perceptions of a research capacity development project for nurses, midwives and allied health professionals. J Nurs Manag. 2008;16(3):315-326.

36. Ziviani J, Wilkinson SA, Hinchliffe F, Feeney R. Mapping allied health evidence-based practice: providing a basis for organisational realignment. Aust Health Rev. 2015;39(3):295-302.

37. Bennett S, Allen S, Caldwell E, et al. Organisational support for evidence-based practice: occupational therapists perceptions. Aust Occup Ther J. 2016;63(1):9-18.

38. Mortenius H, Baigi A, Palm L, et al. Impact of the organisational culture on primary care staff members' intention to engage in research and development. J Health Organ Manag. 2015;29(2):234-251.

39. Sydney Research. Sydney Research Strategic Plan 2014-2019; 2014: 1-28. Available from: https://www.slhd.nsw.gov.au/sydneyresearch/pdf/ ResearchStrategicPlan2014-19.pdf. Accessed March 22, 2017.

40. Sydney Local Health District. Research Strategic Plan 2012-2017; 2012:1-68. Available from: http://www.slhd.nsw.gov.au/pdfs/ResearchStrategicPlan.pdf. Accessed March 22, 2017.

41. Cooke J, Nancarrow S, Dyas J, Williams M. An evaluation of the 'Designated Research Team' approach to building research capacity in primary care. BMC Fam Pract. 2008;9:37.

42. Snyder DC, Brouwer RN, Ennis CL, et al. Retooling institutional support infrastructure for clinical research. Contemp Clin Trials. 2016;48:139-145

43. Pain K, Hagler P, Warren S. Development of an instrument to evaluate the research orientation of clinical professionals. Can J Rehabil. 1996;9(2):93-100.

44. Pitout $\mathrm{H}$. Research orientation of South African occupational therapists. S Afr J Occup Ther. 2013;43(2):5-11. 
45. Wenke R, Mickan S. The role and impact of research positions within health care settings in allied health: a systematic review. BMC Health Serv Res. 2016;16(1):355.

46. Wenke RJ, Ward EC, Hickman I, Hulcombe J, Phillips R, Mickan S. Allied health research positions: a qualitative evaluation of their impact. Health Res Policy Syst. 2017;15:6.

47. Wenke RJ, Mickan S, Bisset L. A cross sectional observational study of research activity of allied health teams: is there a link with self-reported success, motivators and barriers to undertaking research? BMC Health Serv Res. 2017;17(1):1-10.

48. Stephens D, Taylor N, Leggat SG. Research experience and research interests of allied health professionals. J Allied Health. 2009;38(4):107E-111E.

49. Gardner F, Nunan C. How to develop a research culture in a human services organization: integrating research and practice with service and policy development. Qual Soc Work. 2007;6(3):335-351.
50. Kljakovic M. Developing a teaching research culture for general practice registrars in Australia: a literature review. Asia Pac Fam Med. 2009;8(1):1-7.

51. Cusick A. Practitioner-researchers in occupational therapy. Aust Occup Ther J. 2000;47(1):11-27.

52. Cusick A. The experience of clinician-researchers in occupational therapy. Am J Occup Ther. 2001;55(1):9-18.

53. Wrightson PA, Cross VEM. Integrating research into the culture of allied health professions: the background and a review of issues in the United Kingdom. J Allied Health. 2004;33(2):132-138.

54. Friesen EL, Comino EJ, Reath J, et al. Building research capacity in south-west Sydney through a primary and community health research unit. Aust J Prim Health. 2014;20(1):4-8.

55. Ried K, Fuller J. Building a culture of research dissemination in primary health care: the South Australian experience of supporting the novice researcher. Aust Health Rev. 2005;29(1):6-11. 


\section{Supplementary materials}

Table SI Individual research activities and provisions made to perform research from the RCCT

\begin{tabular}{|c|c|c|}
\hline \multicolumn{2}{|c|}{ (A) Individual research activities undertaken in the past 12 months $(n=250)$} & \multirow{2}{*}{$\begin{array}{l}\text { \% Yes } \\
24\end{array}$} \\
\hline $\mathrm{i}$ & Written a research protocol & \\
\hline ii & Submitted an ethics application & 21 \\
\hline iii & Collected data, eg, surveys and interviews & 66 \\
\hline iv & Analyzed qualitative research data & 34 \\
\hline$v$ & Analyzed quantitative research data & 41 \\
\hline vi & Written a literature review & 22 \\
\hline vii & Written a research report or presentation & 39 \\
\hline viii & Presented research findings at a conference in oral presentation or poster format & 23 \\
\hline ix & Presented research findings at conference(s) in more than one presentation or poster & 11 \\
\hline $\mathrm{x}$ & Written a research paper for publication in a journal & 12 \\
\hline xi & Successfully published a research paper in a journal & 10 \\
\hline xii & Successfully published more than one research paper in a journal & 3 \\
\hline xiii & Applied for research funding & 8 \\
\hline xiv & Successfully obtained research funding & 4 \\
\hline$x v$ & Supervised others to undertake a research project & 12 \\
\hline xvi & Provided training to colleagues/staff on research methods & 10 \\
\hline xvii & Formed a new research collaboration with other health professionals & 18 \\
\hline \multicolumn{2}{|c|}{ (B) Research-related activities and provisions made to conduct research } & $\%$ Yes \\
\hline $\mathrm{i}$ & Individuals reporting that research-related activities were part of their role $(n=250)$ & 32 \\
\hline \multirow[t]{9}{*}{ ii } & Provisions made for those individuals to conduct research $(n=80)$ & \\
\hline & Software & 30 \\
\hline & Research supervision & 30 \\
\hline & Time & 37 \\
\hline & Research funds & 11 \\
\hline & Administrative support & 12 \\
\hline & Training & 22 \\
\hline & Library access & 57 \\
\hline & Others & 11 \\
\hline
\end{tabular}

Abbreviation: RCCT, Research Capacity in Context Tool.

Table S2 Barriers and motivators to research $(n=250)$ from the RCCT

\begin{tabular}{lll}
\hline (A) Barriers to research & $\%$ \\
\hline i & Lack of time for research & 91 \\
ii & Lack of suitable backfill & 50 \\
iii & Other work roles take priority & 83 \\
iv & Lack of funds for research & 43 \\
v & Lack of support from management & 24 \\
vi & Lack access to equipment for research & 29 \\
vii & Lack of administrative support & 39 \\
viii & Lack of software for research & 32 \\
ix & Isolation & 10 \\
x & Lack of library/lnternet access & 6 \\
xi & Not interested in research & 8 \\
xii & Other personal commitments & 28 \\
xiii & Desire for work/life balance & 49 \\
xiv & Lack of coordinated approach to research & 27 \\
xv & Lack of skills for research & 49 \\
xvi & Intimidated by research language & 21 \\
xvii & Intimidated by fear of getting it wrong & 21 \\
xviii & Others & 4 \\
\hline & & $($ Continued)
\end{tabular}


Table S2 (Continued)

\begin{tabular}{lll}
\hline (B) & Motivators to performing research & $\%$ \\
\hline i & To develop skills & 84 \\
ii & Career advancement & 56 \\
iii & Increased job satisfaction & 65 \\
iv & Study or research scholarships available & 16 \\
v & Dedicated time for research & 33 \\
vi & Research written into role description & 16 \\
vii & Colleagues doing research & 28 \\
viii & Mentors available to supervise & 42 \\
ix & Research encouraged by managers & 38 \\
x & Grant funds & 23 \\
xi & Links to universities & 28 \\
xii & Forms part of postgraduate study & 17 \\
xiii & Opportunities to participate at own level & 27 \\
xiv & Problem identified that needs changing & 49 \\
xv & Desire to prove a theory/hunch & 32 \\
xvi & To keep the brain stimulated & 50 \\
xvii & Increased credibility & 37 \\
xviii & Others & 4 \\
\hline
\end{tabular}

Abbreviation: RCCT, Research Capacity in Context Tool.

\section{Publish your work in this journal}

The Journal of Multidisciplinary Healthcare is an international, peerreviewed open-access journal that aims to represent and publish research in healthcare areas delivered by practitioners of different disciplines. This includes studies and reviews conducted by multidisciplinary teams as well as research which evaluates the results or conduct of such teams or health care processes in general. The journal covers a very wide range of areas and welcomes submissions from practitioners at all levels, from all over the world. The manuscript management system is completely online and includes a very quick and fair peer-review system. Visit http://www.dovepress.com/ testimonials.php to read real quotes from published authors. 